\title{
Encoding of Natural Scene Movies by Tonic and Burst Spikes in the Lateral Geniculate Nucleus
}

\author{
Nicholas A. Lesica and Garrett B. Stanley \\ Division of Engineering and Applied Sciences, Harvard University, Cambridge, Massachusetts 02138
}

The role of the lateral geniculate nucleus (LGN) of the thalamus in visual encoding remains an open question. Here, we characterize the function of tonic and burst spikes in cat LGN X-cells in signaling features of natural stimuli. A significant increase in bursting was observed during natural stimulation (relative to white noise stimulation) and was linked to the strong correlation structure of the natural scene movies. Burst responses were triggered by specific stimulus events consisting of a prolonged inhibitory stimulus, followed by an excitatory stimulus, such as the movement of an object into the receptive field. LGN responses to natural scene movies were predicted using an integrate-and-fire (IF) framework and compared with experimentally observed responses. The standard IF model successfully predicted LGN responses to natural scene movies during tonic firing, indicating a linear relationship between stimulus and response. However, the IF model typically underpredicted the LGN response during periods of bursting, indicating a nonlinear amplification of the stimulus in the actual response. The addition of a burst mechanism to the IF model was necessary to accurately predict the entire LGN response. These results suggest that LGN bursts are an important part of the neural code, providing a nonlinear amplification of stimulus features that are typical of the natural environment.

Key words: LGN; bursts; tonic; natural scenes; neural coding; integrate and fire

\section{Introduction}

The lateral geniculate nucleus (LGN) of the thalamus is the gateway to the visual cortex, controlling the flow of visual information from the retina [for a review of LGN function, see Sherman (2001a)]. Understanding the neural code of the LGN is an essential first step in characterizing the processing of visual information in higher-level neurons. In the cat, the firing rate of LGN $\mathrm{X}$-cells is linearly related to modulations in the visual stimulus during tonic firing (Guido et al., 1992, 1995). However, after prolonged periods of hyperpolarization, voltage-dependent calcium channels are de-inactivated, and subsequent depolarization of the membrane results in a slow calcium spike that in turn causes a stereotyped burst of closely spaced action potentials (Scharfman et al., 1990; Lu et al., 1992). LGN bursts were first reported as part of rhythmic firing during periods of sleep or low arousal (Livingstone and Hubel, 1981; Steriade et al., 1993). However, a number of studies have since reported LGN bursts interspersed with tonic firing in awake animals and humans (Guido and Weyand, 1995; Radhakrishnan et al., 1999; Ramcharan et al., 2000; Martinez-Conde et al., 2002).

Burst events were originally thought to result from sources unrelated to the visual stimulus. However, recent studies have demonstrated that burst events do indeed encode stimulus-

Received July 27, 2004; revised 0ct. 13, 2004; accepted 0ct. 14, 2004.

This work was supported in part by National Geospatial-Intelligence Agency Grant HM1582-05-C-0009. We thank Yang Dan for the use of the thalamic data and helpful discussion and Markus Meister and Pam Reinagel for useful comments during the later phase of this work.

Correspondence should be addressed to Dr. Garrett B. Stanley, Division of Engineering and Applied Sciences, Harvard University, 321 Pierce Hall, 29 0xford Street, Cambridge, MA 02138. E-mail: gstanley@deas.harvard.edu. DOI:10.1523/JNEUROSCI.3059-04.2004

Copyright $\odot 2004$ Society for Neuroscience ～0270-6474/04/2410731-10\$15.00/0 related information and that the percentage of bursts in the LGN response can be modulated by the statistical properties of the stimulus (Reinagel et al., 1999; Denning et al., 2003; Rivadulla et al., 2003). The relationship between the visual stimulus and the burst response is highly nonlinear, as demonstrated by observing the ratio of the first harmonic to the mean $(F 1 / F 0)$ and frequency-dependent transfer characteristics in LGN responses to drifting gratings during prolonged hyperpolarization (Guido et al., 1995; Mukherjee and Kaplan, 1995). It has been suggested that bursts serve to signal the appearance of a salient stimulus (detection), whereas tonic firing relays detailed features of the stimulus (transmission) (Crick, 1984; Guido et al., 1995). A study of LGN responses in awake behaving cats showed that the response to a drifting grating was characterized by an initial period of bursting at the onset of the stimulus, followed by tonic firing for the duration of the stimulus (Guido and Weyand, 1995). This implies that bursts may serve as a wake-up call, alerting the visual cortex to the presence of a stimulus in the receptive field (RF) and signaling the beginning of tonic relay (Sherman, 2001b). Indeed, bursts were shown to be more effective than tonic spikes at eliciting responses in their cortical targets (Swadlow and Gusev, 2001). Although the results of these studies suggest that LGN bursts may be an important part of the neural code during natural vision, this issue has not yet been explicitly investigated.

Here, the role of LGN bursts in encoding correlated natural stimuli was investigated by analyzing the responses of LGN neurons to natural scene movies. Across a sample of cat LGN X-cells, a significant increase in bursting was observed during natural scene stimulation (relative to white noise stimulation). Bursts were triggered by specific stimulus features, such as the movement of objects into the RF. The stimulus features preceding 
burst events and tonic spikes were characterized, and the results support the detect/transmit framework described above. Using a cascade of a linear spatiotemporal RF, light adaptation, and integrate-and-fire (IF) spike generation, LGN responses to natural scene movies were predicted and compared with those observed experimentally. The standard IF model provides accurate predictions of LGN responses to natural scene movies during tonic firing but tends to underestimate the response during periods of bursting. The addition of a nonlinear amplification mechanism to the IF model is necessary to accurately predict the LGN response during these intervals. These results suggest that LGN bursts may be an important part of the neural code of the LGN, providing an amplification of stimulus features that are typical of correlated natural scenes.

\section{Materials and Methods}

Recordings from cat $L G N$. The data presented here were collected for a previous study, and the experimental preparation has been detailed previously (Stanley et al., 1999). Briefly, adult cats were initially anesthetized with isoflurane ( $3 \%$; with oxygen), followed by sodium pentothal (10 $\mathrm{mg} / \mathrm{kg}$, i.v.; supplemented as needed). A craniotomy $\left(0.5 \mathrm{~cm}^{2}\right)$ was performed over the LGN, and the underlying dura was removed. After surgery, the cats were paralyzed with Norcuron $(0.2 \mathrm{mg} / \mathrm{kg} / \mathrm{hr}$, i.v. $)$ and artificially ventilated. Eyes were refracted, fitted with appropriate contact lenses, and focused on a tangent screen. Recordings were made extracellularly with a multi-electrode array (Eckhorn and Thomas, 1993). Recorded signals were amplified, filtered, and acquired with an analog-todigital board in a personal computer in which spikes were clustered manually. All recordings were made in layer A or A1, and nonlagged $\mathrm{X}$-cells were classified based on the linearity and latency of their responses (Saul and Humphrey, 1990). A total of 37 neurons were included in the original analysis. The final analysis was performed on 18 neurons, the RFs of which were completely within the extent of the white noise stimulus and had a maximal point (see below) that was at least twice the noise level.

Visual stimulation. Visual spatiotemporal stimuli consisting of natural scene movies and white noise were created with a personal computer containing an AT-Vista graphics card (Truevision, Indianapolis, IN) with a monitor refresh rate of $128 \mathrm{~Hz}$. The white noise stimulus was a 4 min binary spatiotemporal m-sequence (Sutter, 1987; Reid et al., 1997). The movies were digitized segments of grayscale video recordings. During movie presentation, the monitor was refreshed at $128 \mathrm{~Hz}$, but the same frame was displayed four times in a row, resulting in an effective frequency of $32 \mathrm{~Hz}$. The movies sampled a range of visual environments, from a series of home video recordings taken in the forest to segments of the Hollywood movie Raiders of the Lost Ark. A full natural scene movie trial for each cell consisted of the entire set of movies (six movies, $16 \mathrm{sec}$ each) repeated eight times. The power-law decay constant of the temporal power spectra averaged over all six movies (and all spatial frequencies) was 2.1 (lowest, 1.6; highest, 2.7), and the power-law decay constant of the spatial power spectra averaged over all six movies (and all temporal frequencies) was 1.9 (lowest, 1.4; highest, 2.5). Spatially, the white noise stimulus consisted of $16 \times 16$ pixels and the natural scene movies consisted of $64 \times 64$ pixels. The pixel size was the same for both stimuli, $0.2^{\circ}$ at $10^{\circ}$ eccentricity. The area of the screen not covered by the white noise stimulus was set to the mean luminance value. The smallest of the stimuli $\left(3.2 \times 3.2^{\circ}\right)$ was significantly larger than the largest RF observed in this study (center plus surround radius of $1.4^{\circ}$ ). The mean luminance of both stimuli was $\sim 40 \mathrm{~cd} / \mathrm{m}^{2}$. The contrast of the stimuli calculated over all pixels and all frames were as follows [Michelson, root mean square (RMS)]: white noise $(1,1)$, movies $(0.71,0.31)$. However, the RMS contrasts of the stimuli after convolution with a typical LGN RF were more similar: 0.32 (noise), 0.21 (movie). This provides a measure of the stimulus contrast in the frequency band relevant to the LGN (Tadmor and Tolhurst, 2000).

Calculation of power spectra. Power spectra were calculated using the Prism interface to the IVE software package (http://www.msg.ucsf.edu/
IVE/). The power spectral density of each stimulus was used to characterize the underlying spatial and temporal correlation structure; a nonuniform concentration of power at lower frequencies is reflective of a high degree of correlation. The power spectrum of each stimulus was calculated using all pixels and all frames, after subtraction of the mean.

Definition of burst events. Bursts were defined according to the standard criterion (Lu et al., 1992; Reinagel et al., 1999). A burst was a group of two or more action potentials, each of which is $<4 \mathrm{msec}$ apart, with the first spike preceded by $>100 \mathrm{msec}$ of silence. Intracellular studies have shown that this criterion is effective for selecting only those spikes originating from calcium-induced bursts (Lu et al., 1992). This is a relatively conservative criterion, intended to minimize the number of detected bursts (Massaux and Edeline, 2003). The results of this study were recalculated using a range of values for the silence $(80-120 \mathrm{msec})$ and maximum spike separation $(4-6 \mathrm{msec})$ times and were not significantly different. Burst identification was based on spike times at $0.1 \mathrm{msec}$ resolution.

Calculation of tonic spike and burst event-triggered averages. Triggered averages were used to determine the average stimulus preceding tonic spikes and burst events (Dayan and Abbott, 2001). The time of a burst event was determined by the time of the first spike in the burst. The 180 msec segments of the stimulus preceding each response event were averaged together. Triggered averages for OFF cells were reflected about the mean luminance for averaging across the sample of cells. For clarity of presentation, these spatiotemporal triggered averages were collapsed to one dimension by averaging across all pixels in the center of the RF for each neuron (as defined below). The results (relative power of the white noise and movie stimuli in the frequency spectra defined by the triggered averages) obtained using these temporal triggered averages were similar to those obtained with the full spatiotemporal triggered averages.

$R F$ estimation. RFs for each cell were estimated from responses to the white noise stimulus using the reverse correlation technique (Marmarelis and Marmarelis, 1978; Dayan and Abbott, 2001). For RF estimation, spike times were binned at $128 \mathrm{~Hz}$ to give an estimate of the firing rate of the neuron. Each spatiotemporal RF estimate consisted of 256 spatial points $(16 \times 16$ grid $)$ spaced at $0.2^{\circ}$ each with 24 temporal points spaced at $7.8 \mathrm{msec}$. The noise in the RF estimate was measured by estimating the $\mathrm{RF}$ for each neuron from randomly shuffled responses. The SD of this estimate (equal to zero in an ideal setting with infinite data) provides a measure of the uncertainty in the actual RF estimate.

Definition of center and surround. RFs were separated into center and surround components using the following method (Reid and Shapley, 2002). First, the point with the largest amplitude (maximal point) in the spatiotemporal RF was determined. Next, the center of the RF was defined as those spatial points at the same latency as the maximal point that (1) formed a contiguous region with the maximal point and other center pixels and (2) had an amplitude with the same sign as the maximal point and a value that was above the error level for that neuron. The surround was defined as a ring around the center region, a maximum of 4 pixels wide.

Prediction of LGN responses. Neural encoding in the LGN can be described using a cascade model including a spatiotemporal integration stage, a light adaptation stage, and an IF spike generation stage (see Fig. $5 a$ ). First, the spatiotemporal stimulus is summed over space and time using the RF of the neuron, estimated from responses to white noise as described above. Next, the signal is high-pass filtered (cutoff, $3 \mathrm{~Hz}$ ) to account for the basic features of retinal light adaptation (Shapley and Enroth-Cugell, 1984; van Hateren et al., 2002). This filtering removes many of the nonstationarities in the natural stimulus and provides a more accurate representation of the input to the LGN. Finally, the signal is scaled (to give an estimate of the stimulus-related input to the neuron) and offset (to compensate for differences in the baseline membrane potential of the model and the actual neuron) and used as the input to a stochastic IF spike generator. The scale and offset parameters were optimized to yield the minimum mean squared error between the actual and predicted response (from the standard IF model described below) to natural scene movies for each cell, using the simplex method (Press et al., 
a

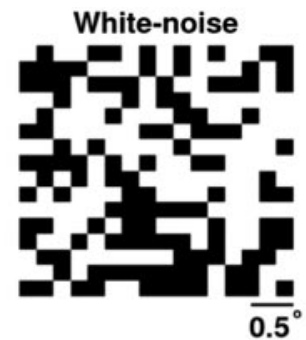

b

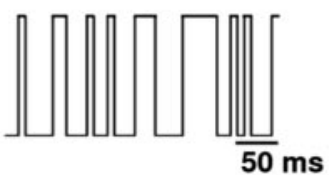

C

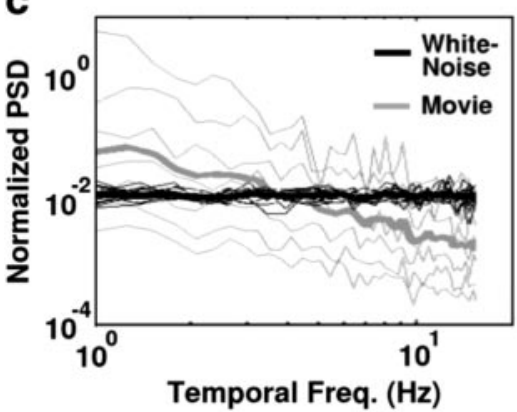

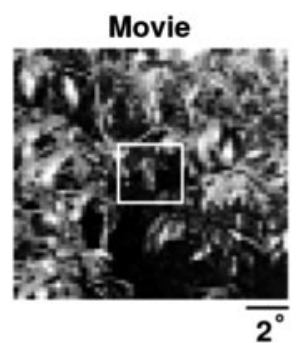
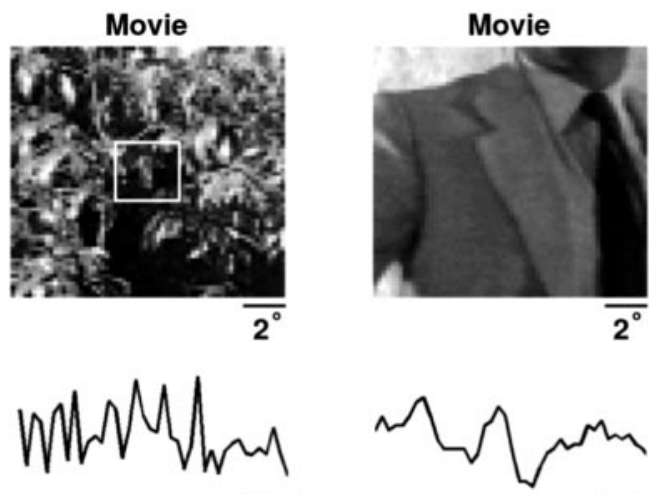

$\overline{200} \mathrm{~ms}$

d

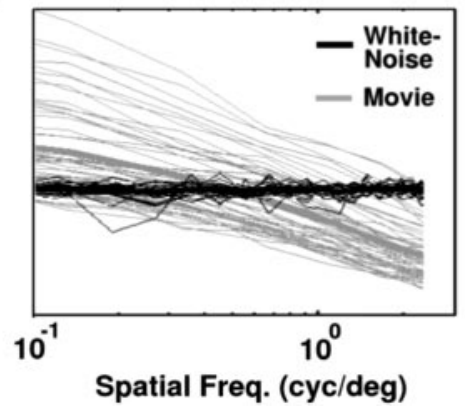

Figure 1. White noise and natural scene movie stimuli. The stimuli used in this study were spatiotemporal white noise and grayscale video recordings. $a$, Sample frames of the stimuli. The white noise (m-sequence) was displayed in a $16 \times 16$ grid. The natural scene movies were displayed in a $64 \times 64 \mathrm{grid}$ that covered the entire screen. The square on the first example frame of the movie indicates an example of the corresponding region of the screen that was covered by the white noise stimulus. The movies included a range of time-varying images, from a set of home video recordings taken in the forest (first example) to Hollywood movies (second example, from the movie Raiders of the Lost Ark). $b$, The temporal variations in the intensity of one pixel of the stimulus. The vertical scale is the same for the white noise and movie stimuli. $c$, The temporal frequency power spectral densities of the white noise (black) and movie (gray) stimuli. Movie spectra were averaged over six different movies. Spectra were normalized so that both stimuli had the same total power. The white noise spectrum was truncated at $16 \mathrm{~Hz}$ for plotting. Spectra were calculated as described in Materials and Methods. The power spectra of spatiotemporal natural scene movies are not space-time separable (Dong and Atick, 1995). Thus, the temporal power spectra are shown at a range of spatial frequencies (thin lines), with the mean across all spatial frequencies denoted by the thick lines. $d$, The spatial frequency power spectra of the white noise and movie stimuli. The spatial power spectra are shown at a range of temporal frequencies (thin lines), with the mean across all temporal frequencies denoted by the thick lines.

1997). The parameters were optimized using the first $48 \mathrm{sec}$ of movie stimulus-response data, whereas the last $48 \mathrm{sec}$ were reserved for testing the predictive power of the optimized model as described below. Optimizing the parameter fit for the prediction of the IF model results in a conservative estimate for the improvement in prediction gained from the addition of the burst mechanism. Many variations on this model were evaluated, and this particular form was chosen as a balance between performance, biophysical plausibility, and simplicity.

The spike generation stage combines a traditional IF model (Knight, 1972 ) with a slow variable representing the de-inactivation of the burstrelated calcium channels. The IF framework used is based on that presented by Smith et al. (2000). IF spike generation (Smith et al., 2000) provides a biologically plausible alternative to the Poisson spike generation commonly used in models of early visual neurons (Keat et al., 2001; Simoncelli et al., 2003; Pillow et al., 2004) and, importantly, can be easily modified to include nonlinear processing mechanisms. Furthermore, the variables in the IF model correspond directly to intracellular quantities, allowing the behavior of the model to be linked directly to physical mechanisms. The IF model used by Smith et al. (2000) was developed from intracellular recordings to predict the response of LGN neurons to sinusoidal current inputs. Here, the model has been adapted to model the extracellular LGN (spiking) response to visual stimuli.
The model is described by the following equations:

$$
\begin{aligned}
C \frac{d V}{d t} & =I_{\mathrm{s}}-I_{\mathrm{L}}-I_{\mathrm{T}}, \\
I_{\mathrm{L}} & =g_{\mathrm{L}}\left(V-V_{\mathrm{L}}\right), \\
I_{\mathrm{T}} & =g_{\mathrm{T}} m_{\infty} h\left(V-V_{\mathrm{T}}\right), \\
\frac{d h}{d t} & =\left\{\begin{array}{cc}
-h / \tau_{\mathrm{h}}^{-}, & \left(V>V_{\mathrm{h}}\right), \\
(1-h) / \tau_{\mathrm{h}}^{+}, & \left(V<V_{\mathrm{h}}\right),
\end{array}\right.
\end{aligned}
$$

where $C$ is the membrane capacitance, $V$ is the membrane potential, $I_{\mathrm{s}}$ is the input current produced by the filtered stimulus, $I_{\mathrm{L}}$ is the leakage current (assuming constant conductance), and $I_{\mathrm{T}}$ is the burst-related calcium current. The activation of the calcium current is controlled by $m_{\infty}=H\left(\mathrm{~V}-V_{\mathrm{h}}\right)$, where $H$ is the Heaviside step function. The dynamics of the calcium current are controlled by $\tau_{\mathrm{h}}^{-}$, which sets the duration of hyperpolarization necessary to activate the burst mechanisms, and $\tau_{\mathrm{h}}^{+}$, which sets the duration of the burst. Model parameters were identical to those used by Smith et al. (2000): $V_{\mathrm{L}}=-65 \mathrm{mV}, C=2 \mu \mathrm{F} / \mathrm{cm}^{2}, g_{\mathrm{L}}=0.035 \mathrm{mS} /$ $\mathrm{cm}^{2}, V_{\text {RESET }}=-50 \mathrm{mV}, V_{\mathrm{h}}=-60 \mathrm{mV}, V_{\mathrm{T}}=$ $120 \mathrm{mV}, \tau_{\mathrm{h}}^{-}=20 \mathrm{msec}, \tau_{\mathrm{h}}^{+}=100 \mathrm{msec}$, and $g_{\mathrm{T}}=0.07 \mathrm{mS} / \mathrm{cm}^{2}$. Although fitting the parameters individually to the response of each neuron may have improved prediction, these values were used to maintain the generality of the model. The model was made stochastic by adding Gaussian noise with zero mean and a SD of $5 \mathrm{mV}$ to the membrane potential. This value was chosen so that the variability (measured as signal/noise ratio) of the model responses matched that observed experimentally. To predict the LGN response with bursts, the integrate-and-fire or burst (IFB) model was used as described above. To predict the LGN response with the standard IF model, the calcium current $I_{\mathrm{T}}$ was set to zero.

For each neuron, predicted responses to eight repeats of the natural scene movie (a 48 sec segment that was not used for model fitting) were generated with the IF and IFB models. The predicted and actual responses were binned at $128 \mathrm{~Hz}$ and averaged across repeats for comparison. Error bars on response predictions were generated using the RF estimate noise as defined above. First, it was assumed that the noise in each RF estimate was distributed normally with zero mean and SD given by the estimate noise. Then for each cell, the distributions of the RF estimate were used to generate 25 different RF realizations, and each instance was used to predict the response of the neuron. A correlation coefficient was calculated between each predicted response and the experimentally observed response for that neuron, and a distribution of correlation coefficients was formed. The error bars on the correlation coefficients in Figure 6 represent one SD of this distribution. When creating the two-dimensional histograms comparing the predicted and actual responses in Figure 6, the actual response during burst intervals was compared with the maximum predicted response in a 24 msec window, so that the predictions were not penalized for a slight temporal offset.

\section{Results}

\section{Response properties of LGN neurons}

Single-unit responses to white noise and natural scene movie stimuli were recorded extracellularly in the LGN of anesthetized cats using a multi-electrode array. Examples of the stimuli are 
shown in Figure 1. Example frames from each stimulus are shown (Fig. 1a) along with the temporal variations in luminance for a typical pixel during the period surrounding the corresponding example frames (Fig. 1b). The different statistical properties of the white noise and natural scene movies are evident in the power spectral densities shown in Figure $1, c$ and $d$. Because the power spectra of the natural scene movies are not space-time separable (Dong and Atick, 1995), the temporal frequency spectra in Figure $1 c$ are shown at a range of spatial frequencies (thin lines), along with the mean across all spatial frequencies (thick lines). The uncorrelated white noise stimulus (black) contains approximately equal power at all temporal frequencies, whereas the natural scene movies (gray), which contain strong correlations, have power spectra that decrease with increasing temporal frequency (Field, 1987; Dong and Atick, 1995). The movie spectra shown are the average over the six movie segments used in this study (96 sec total), which have a range of statistical properties (see Materials and Methods). Similar properties are observable in the spatial frequency spectra shown in Figure $1 d$.

LGN responses were separated into burst and tonic components as illustrated in Figure $2 a$. The figure shows a raster plot of responses to eight repeats of a $250 \mathrm{msec}$ segment of natural scene movie for a typical neuron. Spikes that were part of burst events are shown in gray, and tonic spikes are shown in black. For an event to be classified as a burst, the event had to contain two or more spikes, each $<4 \mathrm{msec}$ apart, preceded by $100 \mathrm{msec}$ of silence (Reinagel et al., 1999). Intracellular studies have shown this to be a stringent criterion for selecting only those spikes originating from calcium-induced bursts (Lu et al., 1992). A range of values for the maximum spike separation and silence times yielded similar results (see Materials and Methods).

A scatter plot comparing the mean firing rates in response to white noise and movie stimuli for each neuron is shown in Figure $2 b$. The mean firing rates for the sample of 18 neurons under white noise and natural stimulation were not significantly different (white noise, $18.6 \pm 5.1 \mathrm{~Hz}$; movie, $17.2 \pm 4.9 \mathrm{~Hz}$; paired $t$ test; $p>0.2$ ). To quantify the level of bursting in the response to each stimulus, the percentage of all spikes that were part of burst events was determined. Figure $2 c$ shows a scatter plot of the burst percentage during white noise and natural stimulation. The burst percentage of movie responses was greater than that of white noise responses in nearly all neurons. The increase in burst percentage during natural stimulation was significant across the sample of cells (white noise, $13.4 \pm$ $6.3 \%$, movie, $25.5 \pm 11.1 \%$; paired $t$ test; $p<0.05$ ).

The increased burst percentage observed during natural stimulation is reflected in the interspike interval (ISI) histograms of the responses shown in Figure $2 d$. The scatter plots show successive ISI pairs for 1000 spikes from the white noise and movie responses of a typical neuron. Although the ISIs in the white noise response are distributed relatively evenly across the histogram, the ISIs in the movie response are clustered in the boxes denoted by the solid lines. The right box denotes those spikes that are potentially the first in a burst event (previous ISI $>100 \mathrm{msec}$, next ISI $<4 \mathrm{msec}$ ), and the left box denotes those spikes that are potentially secondary spikes in a burst event (previous ISI $<4$ msec, next ISI $<4 \mathrm{msec}$ ).

The responses were analyzed further to investigate which properties of the neural response were underlying the higher burst percentage observed during natural stimulation. The average number of spikes per burst event in the sample of LGN cells was similar under white noise and natural stimulation (white noise, $2.67 \pm 0.21$; movie, $2.81 \pm 0.19$; paired $t$ test; $p>0.2$ ). Given that the number of spikes per burst is approximately the
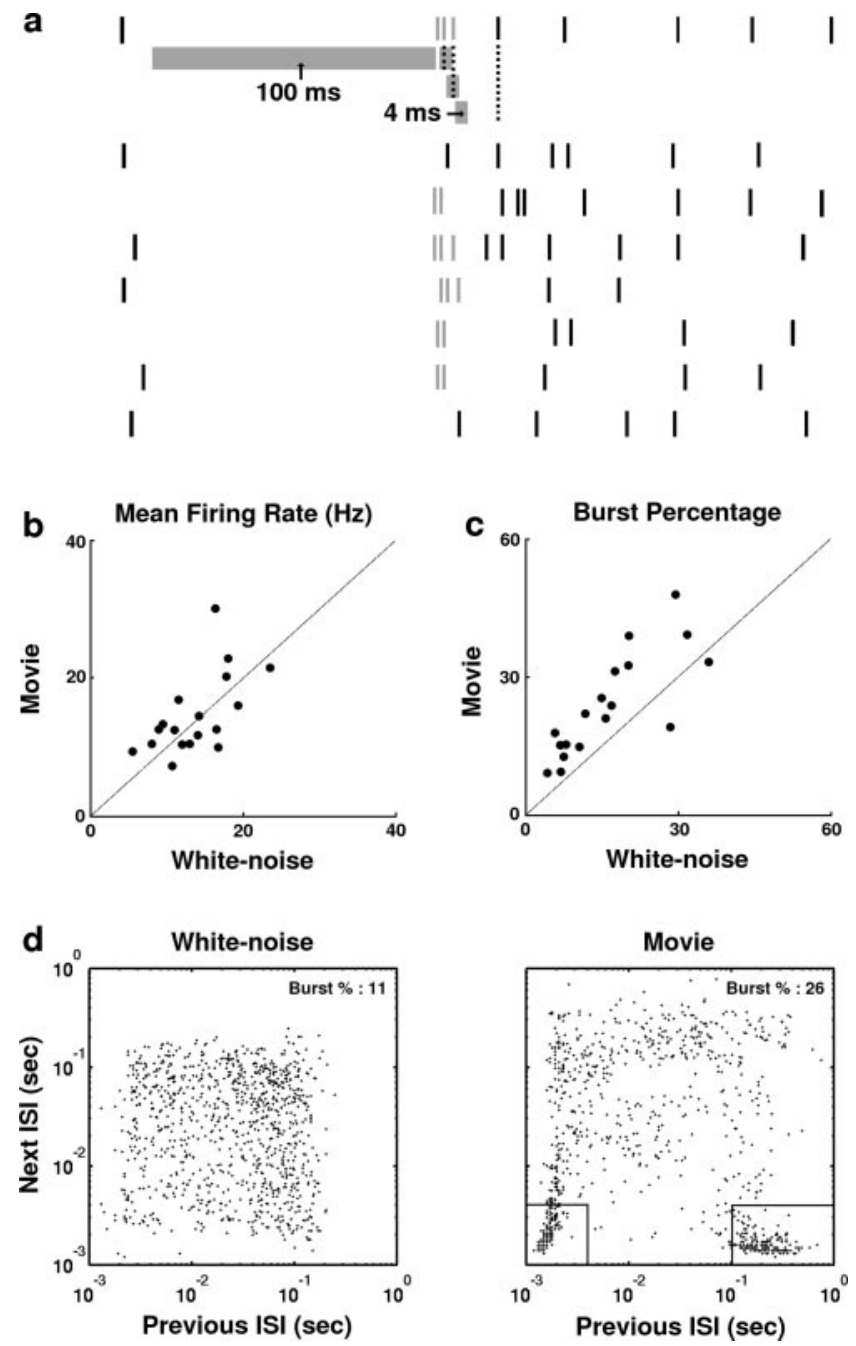

Figure 2. The responses of $L G N$ neurons to white noise and movie stimuli. $a$, A raster plot containing the responses of a typical LGN X-cell to eight repeats of a 250 msec segment of the natural scene stimulus. Responses were separated into tonic and burst components according to an ISI criterion. For a response event to be classified as a burst, it must be preceded by at least 100 msec of silence (no spikes in the interval denoted by the long gray band under first spike train). The event must also contain at least two spikes, separated by $<4$ msec each (successive spikes must fall within the intervals denoted by short gray bands under first spike train). Spikes belonging to burst events are gray, whereas tonic spikes are black. $b$, A scatter plot of the mean firing rates for the sample of $18 \mathrm{LGN}$ cells in response to white noise and movie stimuli. c, A scatter plot of the percentage of white noise and movie responses belonging to burst events for the sample of $18 \mathrm{LGN}$ cells. d, Scatter plots showing the distribution of successive ISIs from 1000 successive spikes in the responses of an LGN neuron to the white noise and movie stimuli. The intervals denoted by black lines indicate candidate burst spikes, as described in Results.

same, there are two other possible sources of the increased level of bursting observed during natural stimulation: the occurrence of more ISIs $>100 \mathrm{msec}$ or the increased likelihood of an ISI $>100$ msec resulting in a burst. Analysis of white noise and movie responses across the sample of LGN cells revealed that both of these factors contributed to the increased bursting during natural stimulation. On average, responses to the natural stimulus exhibited relatively more ISIs $>100 \mathrm{msec}$ (white noise, $24.4 \pm 5.9 \%$ of all ISIs; movie, $30.1 \pm 6.3 \%$ of all ISIs; paired $t$ test; $p<0.05$ ). The percentage of long ISIs that resulted in a burst was also significantly higher during natural stimulation (white noise, $35.2 \pm$ $6.4 \%$; movie, $27.1 \pm 5.1 \%$; paired $t$ test; $p<0.05$ ). These results are consistent with observations first reported in a preliminary study by Denning et al. (2003). 
a

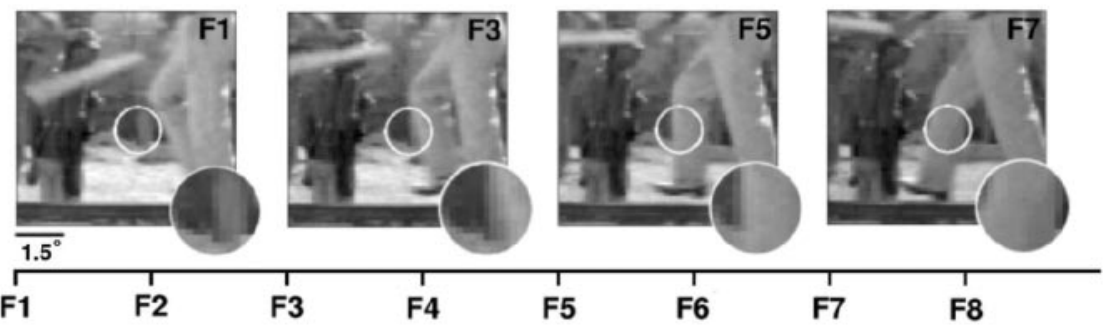

b
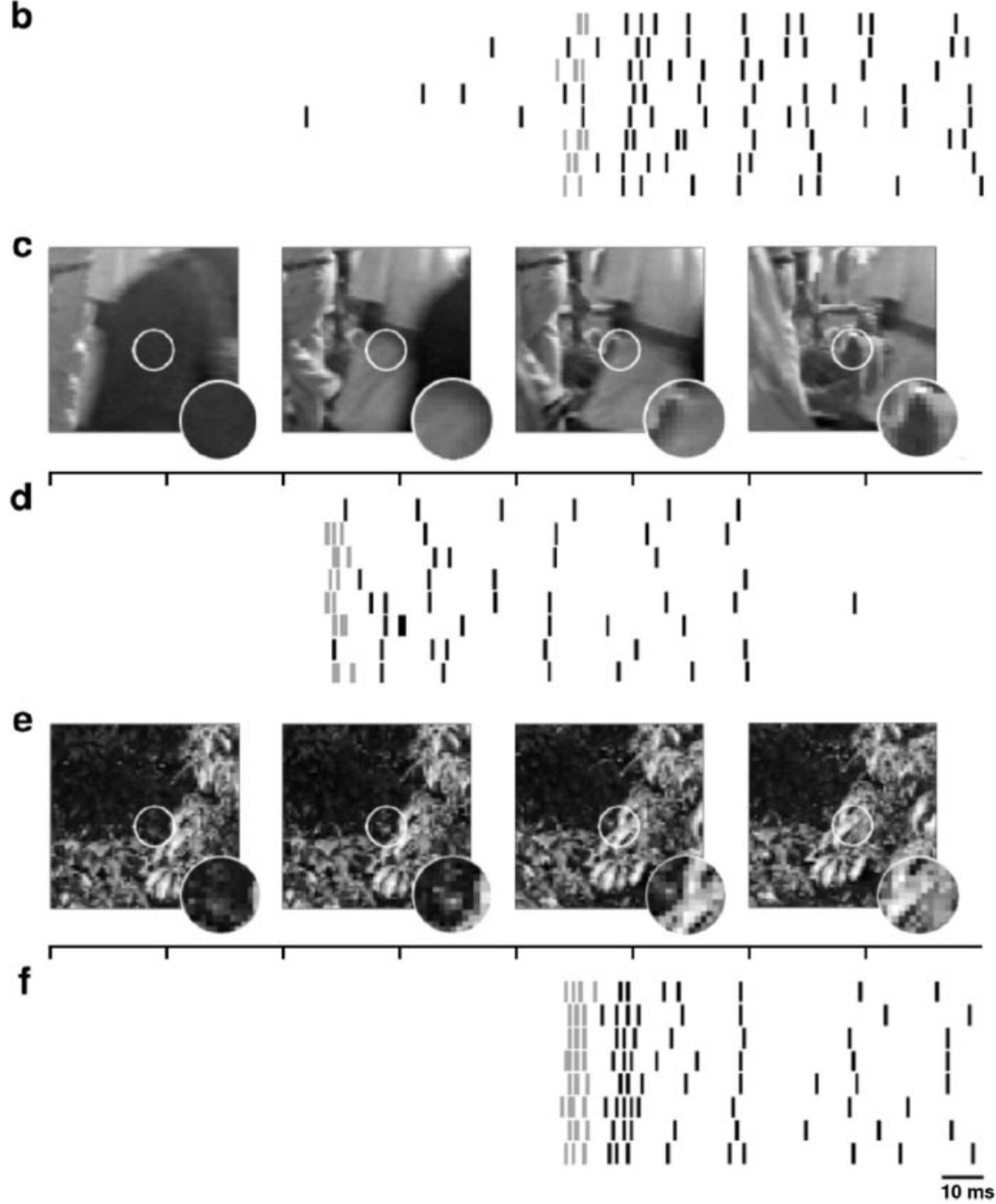

Figure 3. Burst events are triggered by the appearance of objects during natural stimulation. $a$, Frames 1, 3, 5, and 7 of an eight-frame $(256 \mathrm{msec}$ ) sequence of the natural scene movie stimulus corresponding to the responses in $b$. The white circle indicates the RF of the neuron, the responses of which are shown in $b . A 48 \times 48$ pixel region of the entire stimulus for each frame is shown. The inset shows the magnified stimulus inside the RF center. The timeline indicates the onset of each frame (F1-F8). $b$, A raster plot of the response of a typical neuron to eight repeats of the stimulus shown in $a$. Spikes that are part of burst events are gray. $c-f$, Two other stimulus-response pairs for this neuron. Again, for each eight-frame $(256 \mathrm{msec})$ sequence of the stimulus, frames $1,3,5$, and 7 are shown.

\section{Different stimulus features elicit burst and tonic responses}

The responses were separated into tonic and burst components to determine whether specific features of the visual stimulus were leading to the increased bursting observed in the natural scene movie responses. The detect/transmit hypothesis suggests that while the stimulus in the RF of an ON cell is dark, the cell is silent and the membrane is hyperpolarized, setting up the conditions for a burst response. When a bright stimulus enters the RF, a burst is triggered, followed by tonic firing while the stimulus remains in the RF. Indeed, this stimulus-response pattern was observed in the LGN responses to natural scene movies, as shown for a typical ON cell in Figure 3.

Figure $3 a$ shows frames 1, 3, 5, and 7 of an eight-frame $(256 \mathrm{msec})$ sequence of a natural scene movie that elicited the response shown in Figure $3 b$. The timeline in Figure $3 a$ shows the onset of each frame of the stimulus relative to the response in Figure $3 b$. The white circle denotes the RF (center and surround) of the neuron, the responses of which are shown in Figure 3b, and the stimulus inside the RF is magnified in the inset. During the first four frames of the sequence, while the stimulus inside the $\mathrm{RF}$ is dark, the cell is silent. When the bright object enters the RF of the cell in frame 5, a burst is triggered (in five of eight trials), and tonic firing ensues while the object remains in the RF. Similar results are shown in Figure $3, c-f$. Note the reliability of the burst responses [six of eight trials (Fig. $3 d$ ); eight of eight trials (Fig. 3f)].

To quantify this effect across the sample of LGN cells, the temporal average of the stimulus in the RF center preceding tonic spikes and burst events in white noise responses was computed. These triggered averages provide a characterization of the stimulus features that selectively elicit tonic and burst responses. Responses to white noise were used so that the tonic spike and burst event-triggered averages were not contaminated by the correlation structure of the stimulus. Figure $4 a$ shows the tonic spike-triggered average stimulus (black) and the burst event-triggered average stimulus (gray) for the sample of 18 cells (OFF cell averages were reflected about the mean luminance).

It is clear that, on average, the stimuli preceding tonic spikes and burst events have a different statistical structure. The difference is evident in the $100 \mathrm{msec}$ of stimulus that precedes the excitatory rise that triggers the response event. The average stimulus in this time window (from 150 to $50 \mathrm{msec}$ before the response) leading to a tonic spike is flat and close to the mean luminance, reflecting the equal likelihood of bright and dark stimuli in the period preceding the excitatory rise. The average stimulus preceding a burst event, however, decreases steadily and reaches a point well below the mean luminance, indicating the strong likelihood of a prolonged period of darkness (or brightness for an OFF cell) preceding the triggering of a burst event. This difference is not surprising because burst events can only occur after a prolonged hyperpolarization has deinactivated the voltage-dependent calcium channels involved in burst generation. Note also the shorter latency in the triggering of 
the burst event, which results, presumably, from the fact that the calcium spikes that underlie the burst events are triggered at a lower membrane potential threshold than tonic spikes (Zhan et al., 1999).

The power spectra of the triggered averages are shown in Figure $4 b$. The power in the burst-triggered average (gray) is concentrated at low frequencies, indicating that bursts are triggered by those stimulus segments with relatively strong low-frequency content (and thus, strong temporal correlations), whereas the power in the tonic spike-triggered average (black) is more evenly distributed. The power in the white noise and movie stimuli in the frequency ranges in which burst events and tonic spikes are selectively triggered can be quantified by calculating spectral products (multiplying the power spectra of each stimulus by the power spectra of each triggered average and summing the result over all frequencies). The power spectra of the white noise and movie stimuli are shown in Figure $4 c$ (after summation across the same pixels used in the calculation of the triggered averages for each neuron). As expected, the spectrum of the white noise stimulus (dashed) is relatively flat, whereas the spectrum of the movie stimulus (solid) falls off with increasing frequency, because of its strong temporal correlations. Before calculating the spectral products of the stimuli and triggered averages, the spectrum of each triggered average was normalized to have a maximum value of 1 , and the spectrum of each stimulus was normalized to have a total power of 1 , so that the maximum possible value of a given spectral product was 1 . These normalizations allow for a more accurate comparison of the input to the LGN under the two stimulus conditions, because the total power in the retinal output is held relatively constant across a wide range of stimuli by adaptive mechanisms (Shapley and Victor, 1979; Shapley and Enroth-Cugell, 1984). As a result of these normalizations, any difference in the spectral products of the two stimuli and a given triggered average was determined entirely by the relative frequency content of the stimuli. The spectral product of the movie stimulus and burst-triggered average $(0.71)$ was much larger than that of the white noise and burst-triggered average (0.34), reflecting the concentration of the power of the movie stimulus in the low-frequency range in which burst events are selectively triggered. The difference in the spectral products of the two stimuli and the tonic spike-triggered average was much smaller (movie, 0.32; white noise, 0.22 ) because tonic spikes are triggered by stimuli with a broad range of frequencies, including high frequencies at which the power in the movie stimulus has fallen below that of the white noise. These results suggest that the increased bursting observed during natural stimulation is a result of the concentration of the power of the movie stimulus at those low frequencies that selectively trigger burst responses.

\section{Predicted responses to natural scene movies}

LGN X-cells are often assumed to function as linear encoders. This implies that downstream cortical neurons can decode the LGN response by assuming that modulations in firing rate are proportional to intensity fluctuations in the stimulus. However,

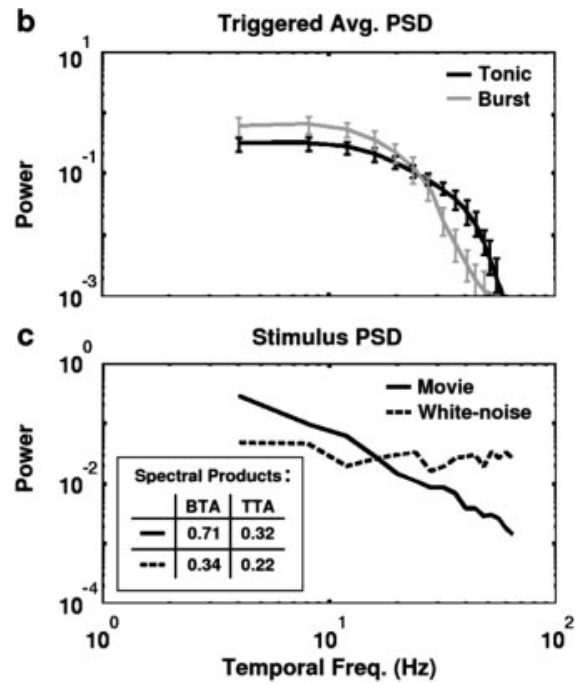

b

Figure 4. Burst events and tonic spikes are triggered by different stimulus features. $a$, The temporal evolution of the average white noise stimulus (triggered average) in the center of the RF preceding burst events (gray) and tonic spikes (black) for a sample (black) shown in a.c, The power spectra of the white noise (dashed) and natural scene movie (solid) stimuli after averaging over all pixels in the center of the RF for each neuron. The spectral products of the normalized stimuli and burst- and tonic-triggered averages (denoted BTA and TTA, respectively) are given in the inset.

the stereotyped nature of burst events results in the nonlinear amplification of a wide range of stimuli to the same neural response. Consequently, the firing rate elicited by a given stimulus in a burst event can far exceed that elicited by the same stimulus during tonic firing. The effect of this nonlinearity in the encoding of natural scene movies can be investigated by predicting the LGN response and comparing the prediction with the actual response. Spatiotemporal RFs were estimated from white noise responses and used in an IF framework (see Materials and Methods) (Fig. 5a) to predict LGN responses to natural scene movies. Briefly, natural scene movies were passed through the spatiotemporal RF of each neuron and passed through an adaptation stage to account for the basic light adaptation properties of retinal processing. The filtered stimulus was scaled to produce a signal reflecting the retinal input to each LGN neuron. The retinal input was used to drive a stochastic IF neuron model, with (IFB) and without (IF) the addition of a voltage-dependent calcium current to generate bursts. For each neuron, a set of eight predicted response repeats were generated to reflect the experimental data.

Examples of a $500 \mathrm{msec}$ segment of experimental and predicted responses for a typical cell are shown in Figure 5. The predicted response to a single stimulus trial for a typical neuron is shown for the IF and IFB models in Figure $5 b$. Raster plots of the predicted and actual responses to eight repeats of the stimulus are shown in Figure $5 c$. Figure $5 d$ shows histograms of the average of the eight repeats of the predicted and actual responses. Intervals containing bursts on two or more trials in the actual response are indicated by asterisks below the histogram in Figure $5 d$. The response events marked by arrows illustrate several properties of the IF and IFB predictions. Event 1 illustrates the tendency of the IF model (solid black) to underpredict the actual response (gray) during burst events. Although the IF prediction is linearly related to the stimulus intensity, the burst response of the actual neuron represents a nonlinear amplification. The additional bursting mechanism in the IFB model (dashed black) captures this amplification. As illustrated by event 2, certain response events are 
a

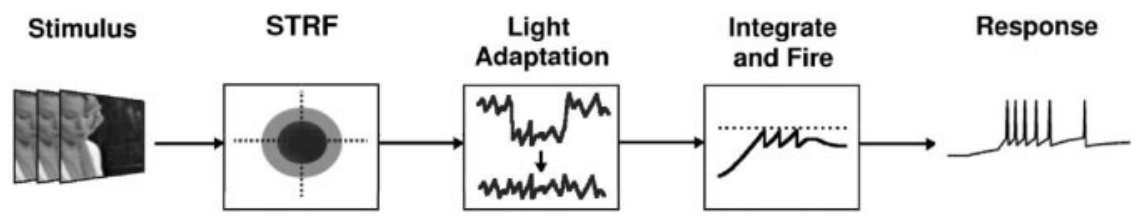

b
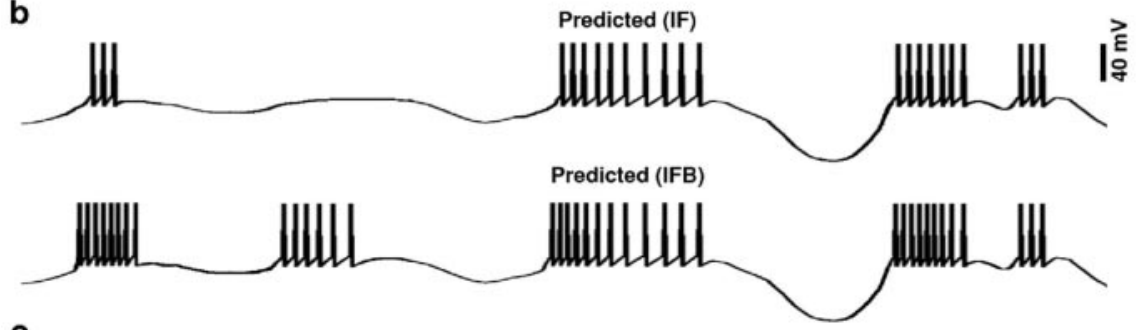

C
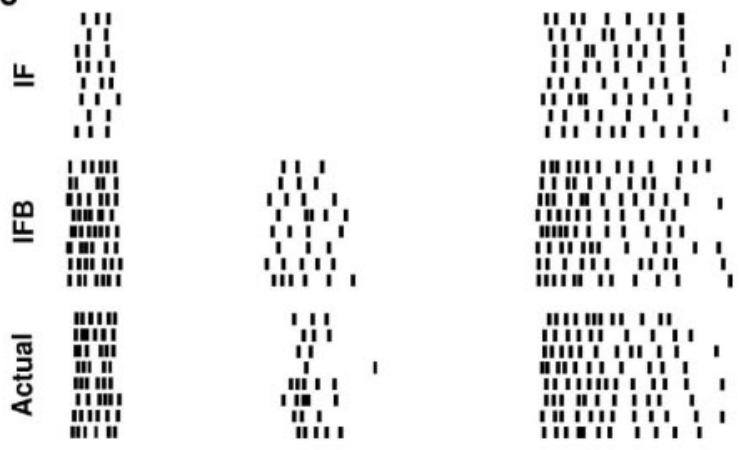

d

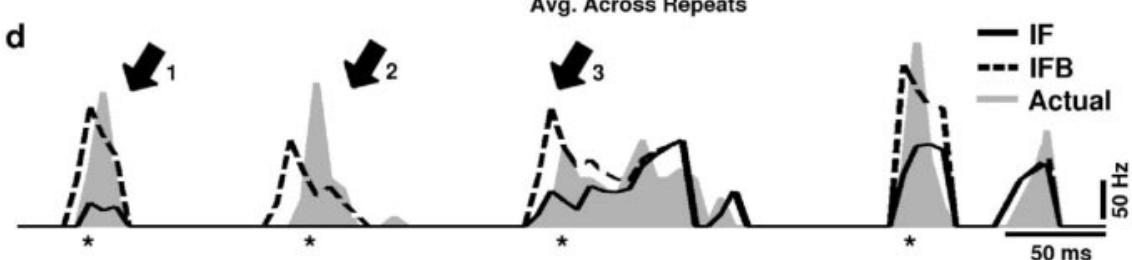

Figure 5. IF predictions of the LGN response to natural scene movies. $a, A$ block diagram of the LGN encoding model. The spatiotemporal natural scene movie is passed through the spatiotemporal RF (STRF) of the neuron, then through a light adaptation stage, and used to drive a stochastic IF or IFB spike generator. $b$, The predicted response to a single trial of a 500 msec segment of the natural scene movie stimulus from the IF model and IFB models for a typical cell. c, A raster plot of the predicted and actual responses to eight repeats of the $500 \mathrm{msec}$ segment of the natural scene movie stimulus. $d$, A histogram of actual and predicted responses to the segment of natural scene movie averaged across eight repeats. The prediction from the IF model is shown in solid black, the prediction from the IFB model is shown in dashed black, and the actual response of the neuron is shown in gray. Intervals in the actual response at which a burst event occurred on two or more trials are indicated by asterisks. Spike times were collected into 7.8 msec bins.

completely missed by the IF model. This is a consequence of the fact that the slow calcium spike underlying the burst event is triggered at a much lower threshold than tonic spikes. Thus, a stimulus that would not normally cause the membrane potential to reach threshold can generate a response if the bursting mechanism is activated. Again, the IFB model accounts for this behavior. Event 3 illustrates the equivalence of the IF and IFB models during tonic firing. Although this firing event starts with a burst, resulting in different predictions from the IF and IFB model, the event continues with tonic spiking that is well predicted by both models.

The behavior illustrated in Figure 5 was consistently observed across the sample of cells. Two-dimensional histograms comparing the predicted and actual responses during tonic and burst intervals over a $48 \mathrm{sec}$ segment of the movie for the IF and IFB models are shown in Figure $6 a$ (for definition of burst and tonic intervals, see figure legend).

During tonic firing, the predicted and actual responses are

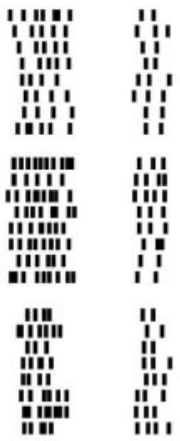

distributed around the line of equality for both models. During burst firing, the IF model consistently underpredicts the LGN response, and the predicted and actual response are poorly matched. In contrast, the actual LGN response and the prediction of the IFB model are well matched, even during intervals of bursting. To illustrate the difference in the predictive power of the IF and IFB models across the sample of LGN cells, correlation coefficients between the predicted and actual responses during tonic and burst intervals were calculated for each cell. As Figure $6 b$ shows, the predictions of the IF and IFB models were similar during tonic firing (mean correlation coefficients: IF $=0.61$, IFB $=$ 0.59 ; paired $t$ test; $p>0.2$ ), whereas during periods of bursting, the IFB model significantly outperformed the IF model (IF $=$ 0.46 , IFB $=0.61$; paired $t$ test; $p<0.05$ ).

\section{Discussion}

The results presented here demonstrate that LGN burst responses are a nonlinear amplification of stimulus features that are typical of correlated natural stimuli. In a sample of 18 LGN X-cells, nearly all exhibited increased bursting in response to the natural stimulus compared with white noise stimulus (Fig. 2c). This increased bursting resulted from both the presence of more long ISIs ( $>100 \mathrm{msec}$ ) and an increase in the probability of a long ISI resulting in a burst during natural stimulation. Burst events were triggered by the presence of a prolonged inhibitory stimulus in the RF, followed by an excitatory stimulus. This was evident both in observing the features of the movie stimulus that elicited burst events (Fig. 3) and in analyzing the average stimulus preceding tonic spikes and burst events under white noise stimulation (Fig. 4). Calculating spectral products revealed that the movie stimulus contained more power in the lowfrequency range in which burst responses are selectively triggered, suggesting that the increased bursting observed during natural stimulation is a result of the strong correlation structure of the movie stimulus.

The role of tonic and burst responses in the neural code of the LGN was assessed by using an IF framework to predict the response of LGN neurons to natural scene movies (Figs. 5, 6). The predicted responses of both the IF and IFB models closely matched the actual response during tonic firing, indicating an approximately linear relationship between stimulus intensity and modulations in firing rate. However, during periods of bursting, this linear relationship is no longer valid because burst responses represent a nonlinear amplification of the visual stimulus. Consequently, the predicted response of the IF model typically underestimated the actual response during periods of bursting, and the additional mechanism in the IFB model was necessary to accurately capture the LGN response during these intervals. 


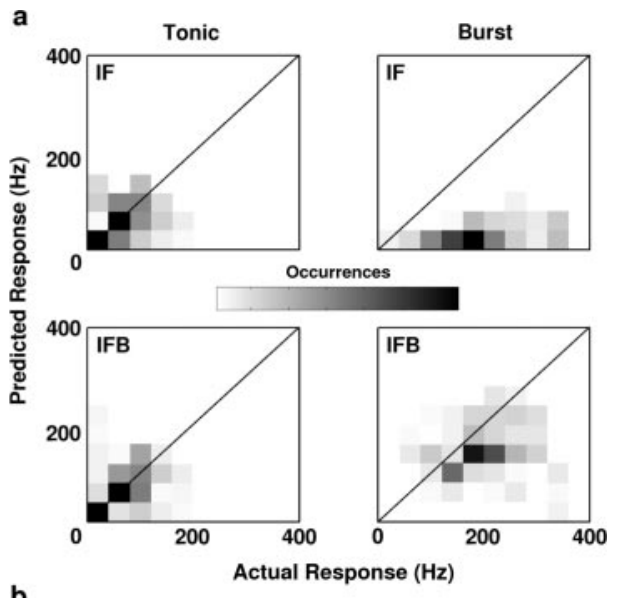

b
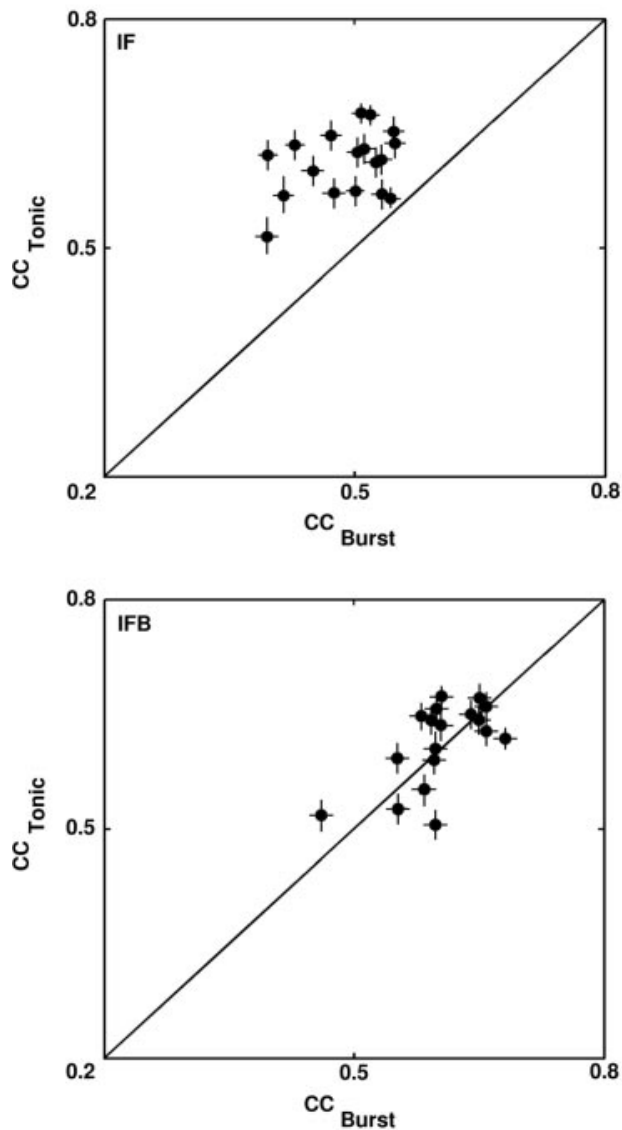

Figure 6. The standard IF model underpredicts the $L G N$ response during periods of bursting. $a, A$ series of two-dimensional histograms comparing the actual and predicted $L G N$ responses during burst and tonic firing intervals for a typical $\mathrm{ON}$ cell (averaged across 8 repeats). Dark areas indicate a large number of occurrences, and light areas indicate a small number of occurrences. Histograms were normalized so that the bin with the largest number of occurrences was shown in black. On each histogram, the equality line is shown. Histograms were calculated using responses to a different $48 \mathrm{sec}$ segment of the natural scene movie from that used to optimize the model parameters. Burstintervals were defined as those intervals during which a burst occurred on at least two of eight trials. Tonic intervals were all other intervals that contained at least one spike on any of the eight trials. Intervals during which no spikes occurred on any of the eight trials were not included in the analysis.b, Scatter plots of the correlation coefficients (CC) between actual and predicted responses to natural scene movies during burst and tonic firing intervals for the sample of 18 LGN X-cells. Coefficients for the IF model are shown in the top plot, and coefficients for the IFB model are shown in the bottom plot. Error bars represent one SD of the distribution of correlation coefficients generated for each neuron, as described in Materials and Methods.
LGN bursts are a more effective means of stimulus detection than tonic spikes because of the increased signal/noise ratio during burst firing (Guido et al., 1995) and the enhanced ability to cause postsynaptic spikes (Swadlow and Gusev, 2001), and have been associated with the onset of a stimulus or eye movements that bring a stimulus into the RF in awake animals (Guido and Weyand, 1995; Martinez-Conde et al., 2002). These results have led to the hypothesis that bursts events serve to detect the presence of a salient stimulus, whereas tonic firing serves to transmit details about stimulus features (Sherman, 2001a). The results presented here show that bursts are triggered by specific features of the visual stimulus that are prominent during natural stimulation. For the sample of LGN X-cells tested here, the average stimulus preceding a burst (for an ON cell) was dark for the 100 msec before the triggering of the response event (150-50 msec before the burst), whereas the average stimulus during the same period preceding a tonic spike was equally likely to be bright or dark. Considering these results in the context of the detect/transmit framework, a possible role for bursting in LGN neurons during natural vision becomes evident. While there is no salient stimulus in the RF, the neuron is silent and membrane is hyperpolarized, optimizing the conditions for stimulus detection. When a stimulus enters the RF (via saccade or movement of an object into the visual field) a burst is triggered, alerting the cortex to its presence and perhaps commanding the allocation of attentional resources. While the stimulus persists in the RF, the mean firing rate of the neuron is increased, and tonic firing relays details to the cortex via linear modulations in firing rate. When the stimulus leaves the RF, the neuron falls silent and the cycle repeats. Indeed, this silence/burst/tonic/repeat response pattern was evident in the LGN responses to natural scene movies, as objects moved in and out of the RF.

Results from other sensory areas have also demonstrated the stimulus specificity of burst events (Lisman, 1997; Krahe and Gabbiani, 2004). Burst events in complex cells of the cat visual cortex have sharper orientation tuning than isolated spikes (Cattaneo et al., 1981). In pyramidal cells in the electrosensory system of weakly electric fish, burst events were shown to be triggered reliably by upstrokes (or downstrokes) of the stimulus (Gabbiani et al., 1996; Metzner et al., 1998; Krahe et al., 2002). Recently, it was demonstrated that these pyramidal burst responses are preferentially triggered by low-frequency stimuli, while isolated spike responses encoded the entire frequency range of the stimulus (Oswald et al., 2004). Similar results have been obtained from modeling studies of bursting pyramidal neurons (Kepecs et al., 2002; Kepecs and Lisman, 2003).

A previous study by Reinagel et al. (1999) using grating stimuli found that the average stimuli preceding burst and tonic responses in the LGN were similar. The difference between the result of Reinagel et al. (1999) and the results presented here is likely to attributable to the choice of stimuli (P. Reinagel, personal communication). In the study by Reinagel et al. (1999), the excitatory phase of a static grating was centered over the RF center, and the spatial contrast of the grating was modulated in time. Consequently, the stimulus in the RF center was always excitatory (never less than the mean luminance of the monitor for an $\mathrm{ON}$ cell), and the conditions that preferentially triggered bursts in the data presented here (prolonged inhibitory stimulus, followed by an excitatory stimulus) were not tested.

Anesthetic levels are known to influence thalamic bursting, potentially increasing the probability of observing low-threshold calcium spikes (Massaux and Edeline, 2003). However, studies have demonstrated that burst events also occur in awake animals 
and are generated in a stimulus-specific manner similar to that demonstrated here. LGN bursts related to the onset of visual stimuli have been observed in awake behaving cats, in which LGN response to a drifting grating was characterized by an initial period of bursting at the onset of the stimulus, followed by tonic firing for the duration of the stimulus (Guido and Weyand, 1995). A number of other studies have also reported LGN bursts interspersed with tonic firing in awake monkeys and humans (Radhakrishnan et al., 1999; Ramcharan et al., 2000; MartinezConde et al., 2002). In the studies of LGN burst responses in awake cats and monkeys, the average burst percentage during visual stimulation was $\sim 10 \%$, which is within, but at the lower end of, the range of burst percentages observed here. In the present study, even those neurons with relatively low burst percentages exhibited nonlinear amplification of specific stimulus features (e.g., the burst percentage of the neuron shown in Figs. 3 and 5 during natural stimulation was $9 \%$ ). Thus, it is likely that the effects observed here would also be present in the awake animal, but, ultimately, this can only be determined by direct investigation.

The results presented here have not addressed the role of control signals from the brainstem or cortex on LGN bursting. It has been shown that the majority of inputs to LGN neurons do not come from the retina (Erisir et al., 1997). During sleep, rhythmic bursting oscillations in the LGN are thought to be controlled by the brainstem (Steriade et al., 1993), whereas bursts observed interspersed with tonic firing in the alert state are thought to be influenced by cortical feedback (Sherman, 2001a; Destexhe and Sejnowski, 2002; Krahe and Gabbiani, 2004). Recently, manipulation of cortical feedback was shown to modulate the proportion of burst and tonic responses in LGN neurons (Wang et al., 2001; Allito et al., 2003). One possible role for cortical feedback during natural vision may be to depolarize thalamic neurons after a burst response, accelerating the switch from burst to tonic firing after the detection of a stimulus and maintaining a high spontaneous firing rate for linear transmission of stimulus features (Sillito and Jones, 2002; Krahe and Gabbiani, 2004).

\section{References}

Allito HJ, Weyand TG, Usrey WM (2003) Corticothalamic interactions and the role of thalamic bursts in visual processing. Soc Neurosci Abstr 29:485:16.

Cattaneo A, Maffei L, Morrone C (1981) Patterns in the discharge of simple and complex visual cortical cells. Proc R Soc Lond B Biol Sci 212:279-297.

Crick F (1984) Function of the thalamic reticular complex: the searchlight hypothesis. Proc Natl Acad Sci USA 81:4586-4590.

Dayan P, Abbott LF (2001) Theoretical neuroscience. Cambridge, MA: MIT.

Denning KS, Reid RC, Reinagel P (2003) LGN burst state is controlled by visual signals. Soc Neurosci Abstr 29:699:12.

Destexhe A, Sejnowski TJ (2002) The initiation of bursts in thalamic neurons and the cortical control of thalamic sensitivity. Proc R Soc Lond B Biol Sci 357:1649-1657.

Dong DW, Atick JJ (1995) Statistics of time-varying images. Network Comput Neural Syst 6:345-358.

Eckhorn R, Thomas U (1993) A new method for the insertion of multiple microprobes into neural and muscular tissue, including fiber electrodes, fine wires, needles, and microsensors. J Neurosci Methods 49:175-179.

Erisir A, Horn SCV, Sherman SM (1997) Relative numbers of cortical and brainstem inputs to the lateral geniculate nucleus. Proc Natl Acad Sci USA 94:1517-1520.

Field DJ (1987) Relations between the statistics of natural images and the response properties of cortical cells. J Opt Soc Am [A] 4:2379-2393.

Gabbiani F, Metzner W, Wessel R, Koch C (1996) From stimulus encoding to feature extraction in weakly electric fish. Nature 384:564-567.

Guido W, Weyand T (1995) Burst responses in thalamic relay cells of the awake behaving cat. J Neurophysiol 74:1782-1786.
Guido W, Lu S, Sherman SM (1992) Relative contributions of burst and tonic responses to the receptive field properties of lateral geniculate neurons in the cat. J Neurophysiol 68:2199-2211.

Guido W, Lu S, Vaughan JW, Godwin DW, Sherman SM (1995) Receiver operating characteristic (ROC) analysis of neurons in the cat's lateral geniculate nucleus during tonic and burst firing mode. Vis Neurosci 12:723-741.

Keat J, Reinagel P, Reid RC, Meister M (2001) Predicting every spike: a model for the responses of visual neurons. Neuron 30:803-817.

Kepecs A, Lisman J (2003) Information encoding and computation with spikes and bursts. Network Comput Neural Syst 14:103-118.

Kepecs A, Wang X, Lisman J (2002) Bursting neurons signal input slope. J Neurosci 22:9053-9062.

Knight BW (1972) Dynamics of encoding in a population of neurons. J Physiol (Lond) 59:734-766.

Krahe R, Gabbiani F (2004) Burst firing in sensory systems. Nat Rev Neurosci 5:13-23.

Krahe R, Kreiman G, Gabbiani F, Koch C, Metzner W (2002) Stimulus encoding and feature extraction by multiple sensory neurons. J Neurosci 22:2374-2382.

Lisman JE (1997) Bursts as a unit of neural information: marking unreliable synapses reliable. Trends Neurosci 20:38-43.

Livingstone MS, Hubel DH (1981) Effects of sleep and arousal on the processing of visual information in the cat. Nature 291:554-561.

Lu SM, Guido W, Sherman SM (1992) Effects of membrane voltage on receptive field properties of lateral geniculate neurons in the cat: contributions of the low-threshold $\mathrm{Ca}^{2[\mathrm{~B} 7]}$ conductance. J Neurophysiol 68:2185-2198.

Marmarelis PZ, Marmarelis VZ (1978) Analysis of physiological systems. New York: Plenum.

Martinez-Conde S, Macknik SL, Hubel DH (2002) The function of bursts of spikes during visual fixation in the awake primate lateral geniculate nucleus and primary visual cortex. Proc Natl Acad Sci USA 99:13920-13925.

Massaux A, Edeline J (2003) Bursts in the medial geniculate body: a comparison between anesthetized and unanesthetized states in guinea pig. Exp Brain Res 153:573-578.

Metzner W, Koch C, Wessel R, Gabbiani F (1998) Feature extraction by burst-like spike patterns in multiple sensory maps. J Neurosci 18:2283-2300.

Mukherjee P, Kaplan E (1995) Dynamics of neurons in the cat lateral geniculate nucleus: in vivo electrophysiology and computational modeling. J Neurophysiol 74:1222-1243.

Oswald AM, Chacron MJ, Doiron B, Batisan J, Maler L (2004) Parallel processing of sensory input by bursts and isolated spikes. J Neurosci 24:4351-4362.

Pillow JW, Paninski L, Simoncelli EP (2004) Maximum likelihood estimation of a stochastic integrate-and-fire neural model. Adv Neural Inform Process Syst 16:1311-1318.

Press W, Teukolsky S, Vetterling W, Flannery B 1997 Numerical recipes in C. New York: Cambridge.

Radhakrishnan V, Tsoukatos J, Davis KD, Tasker RR, Lozano AM, Dostrovsky JO (1999) A comparison of the burst activity of lateral thalamic neurons in chronic pain and non-pain patients. Pain 80:567-575.

Ramcharan EJ, Gnadt JW, Sherman SM (2000) Burst and tonic firing in thalamic cells of unanesthetized, behaving monkeys. Vis Neurosci $17: 55-62$.

Reid RC, Shapley RM (2002) Space and time maps of cone photoreceptor signals in macaque lateral geniculate nucleus. J Neurosci 22:6158-6175.

Reid RC, Victor JD, Shapley RM (1997) The use of m-sequences in the analysis of visual neurons: linear receptive field properties. Vis Neurosci 14:1015-1027.

Reinagel P, Godwin D, Sherman SM, Koch C (1999) Encoding of visual information by LGN bursts. J Neurophysiol 81:2558-2569.

Rivadulla C, Martinez L, Grieve KL, Cudiero J (2003) Receptive field structure of burst and tonic firing in the feline lateral geniculate nucleus. J Physiol (Lond) 553:601-610.

Saul AB, Humphrey AL (1990) Spatial and temporal response properties of lagged and nonlagged cells in cat lateral geniculate-nucleus. J Neurophysiol 64:206-224.

Scharfman HE, Lu SM, Guido W, Adams PR, Sherman MS (1990) $\mathrm{N}$-methyl-D-aspartate receptors contribute to excitatory postsynaptic po- 
tentials of cat lateral geniculate nucleus neurons recorded in thalamic slices. Proc Natl Acad Sci USA 87:4548-4552.

Shapley R, Enroth-Cugell C (1984) Visual adaptation and retinal gain controls. Prog Ret Res 3:263-346.

Shapley RM, Victor JD (1979) The contrast gain control of the cat retina. Vision Res 19:431-434.

Sherman SM (2001a) Tonic and burst firing: dual modes of thalamocortical relay. Trends Neurosci 24:122-126.

Sherman SM (2001b) A wake-up call from the thalamus. Nat Neurosci 4:344-346.

Sillito AM, Jones HE (2002) Corticothalamic interaction in the transfer of visual information. Philos Trans R Soc Lond B Biol Sci 357:1739-1752.

Simoncelli E, Paninsky L, Pillow J, Schwartz O (2003) Characterization of neural responses with stochastic stimuli. In: The cognitive neurosciences, Ed 3 (Gazzaniga M, ed), pp 113-141. Cambridge, MA: MIT.

Smith GD, Cox CL, Sherman SM, Rinzel J (2000) Fourier analysis of sinusoidally driven thalamocortical relay neurons and a minimal integrateand-fire-or-burst model. J Neurophysiol 83:588-610.

Stanley GB, Li FF, Dan Y (1999) Reconstruction of natural scenes from ensemble responses in the lateral geniculate nucleus. J Neurosci 19:8036-8042.

Steriade M, McCormick DA, Sejnowski TJ (1993) Thalamocortical oscillations in the sleeping and aroused brain. Science 262:679-685.

Sutter EE (1987) A practical non-stochastic approach to nonlinear time domain analysis. Adv Methods Physiol Syst Model 1:303-315.

Swadlow HA, Gusev AG (2001) The impact of "bursting" thalamic impulses at a neocortical synapse. Nat Neurosci 4:402-408.

Tadmor Y, Tolhurst DJ (2000) Calculating the contrasts that retinal ganglion cells and LGN neurones encounter in natural scenes. Vision Res 40:3145-3157.

van Hateren JH, Ruttiger L, Sun H, Lee BB (2002) Processing of natural temporal stimuli by macaque retinal ganglion cells. J Neurosci 22:9945-9960.

Wang W, Jones HE, Andiola IM, Salt TE, Sillito AM (2001) Focal activation of feedback from V1 to LGN shifts firing patterns between bursting and tonic modes. Soc Neurosci Abstr 27:723.3.

Zhan XJ, Cox CL, Rinzel J, Sherman SM (1999) Current clamp and modeling studies of low-threshold calcium spikes in cells of the cat's lateral geniculate nucleus. J Neurophysiol 81:2360-2373. 